\title{
The transcription factor Maf-S regulates metabolic resistance to insecticides in the malaria vector Anopheles gambiae
}

\author{
Victoria A. Ingham ${ }^{1 *}$, Patricia Pignatelli ${ }^{1}$, Jonathan D. Moore ${ }^{2}$, Simon Wagstaff ${ }^{1}$ and Hilary Ranson ${ }^{1 *}$ (D
}

\begin{abstract}
Background: Malaria control in Africa is dependent upon the use insecticides but intensive use of a limited number of chemicals has led to resistance in mosquito populations. Increased production of enzymes that detoxify insecticides is one of the most potent resistance mechanisms. Several metabolic enzymes have been implicated in insecticide resistance but the processes controlling their expression have remained largely elusive.

Results: Here, we show that the transcription factor Maf-S regulates expression of multiple detoxification genes, including the key insecticide metabolisers CYP6M2 and GSTD1 in the African malaria vector Anopheles gambiae. Attenuation of this transcription factor through RNAi induced knockdown reduced transcript levels of these effectors and significantly increased mortality after exposure to the pyrethroid insecticides and DDT (permethrin: 9. $2 \%$ to $19.2 \%(p=0.015)$, deltamethrin: $3.9 \%$ to $21.6 \%(p=0.036)$ and DDT: $1 \%$ to $11.7 \%(p=<0.01)$, whilst dramatically decreasing mortality induced by the organophosphate malathion $(79.6 \%$ to $8.0 \%(p=<0.01)$ ). Additional genes regulated by Maf-S were also identified providing new insight into the role of this transcription factor in insects.
\end{abstract}

Conclusion: Maf-S is a key regulator of detoxification genes in Anopheles mosquitoes. Disrupting this transcription factor has opposing effects on the mosquito's response to different insecticide classes providing a mechanistic explanation to the negative cross resistance that has been reported between pyrethroids and organophosphates.

Keywords: Insecticide resistance, Transcriptional control, Cross resistance, Metabolic resistance, Mosquito, Anopheles gambiae

\section{Background}

Insecticides play a key role in controlling malaria vectors and hence preventing disease transmission. An increase in the use of insecticide treated bednets (ITNs) and indoor residual spraying (IRS) has led to a dramatic decrease in malaria cases since 2000 [1]. However, increased exposure to a limited range of insecticides has led to the emergence of resistance to these compounds [2] which poses a serious threat to the future of malaria control efforts. Four major insecticide classes are used to control adult mosquitoes; pyrethroids, DDT, carbamates and organophosphates. All four are used in IRS

\footnotetext{
* Correspondence: victoria.ingham@|stmed.ac.uk; hilary.ranson@|stmed.ac.uk ${ }^{1}$ Liverpool School of Tropical Medicine, Pembroke Place, Liverpool, England, L35QA

Full list of author information is available at the end of the article
}

programmes but all ITNs are treated with pyrethroids [1]. Resistance to all classes has been reported in African malaria vectors with pyrethroid resistance spreading exceptionally rapidly in recent years [2]. Understanding the mechanisms underpinning this resistance is an important prerequisite for the identification of resistance management strategies.

The two best characterised causes of insecticide resistance are target-site resistance and metabolic resistance [3]. Mutations in the voltage-gated sodium channel, the target of both DDT and pyrethroid insecticides, cause the phenotype known as knockdown resistance or $k d r$. Although this resistance mechanism is readily tracked through PCR and therefore widely reported, metabolic resistance has a greater operational impact on malaria control [4]. Metabolic resistance is more complex to elucidate at the molecular level and can involve changes in 
the rate of sequestration, detoxification and/or transport of insecticides or their conjugates. Increased expression of genes involved in each of the detoxification stages has been associated with insecticide resistance in Anopheles mosquitoes [5] but the mechanisms controlling expression of these genes have not been identified.

The transcriptional response to exposure and adaptation to xenobiotics is well studied in mammalian systems and is regulated by three major families, all of which cause induction of phase I, II and III drug metabolism enzymes when activated:, (i) aryl hydrocarbon receptor-aryl hydrocarbon receptor nuclear translocator (ARNT-AhR) [6] (ii) the pregnane $\mathrm{X}$ and constitutive androstane receptors (PXR and CAR) [7] and (iii) (i) Nrf2-Maf [8]. Homologs of these transcriptional regulators have been identified in invertebrates and shown to regulate genes involved in response to xenobiotics. For example, Tango-spineless the homologs of ARNT-AhR, enhance the expression of the cytochrome P450 CYP6B1 from black swallowtail caterpillars in response to allelochemicals [9]. DHR96, the homo$\log$ of the human PXR-CAR receptors, regulates sensitivity to DDT through changes to the expression of the CYP6 family of P450s and other key detoxification families in Drosophila [10]. Lastly the invertebrate equivalent of the Nrf2-Maf pathway in Drosophila, cnc-Maf-S, has been shown to modify resistance to both malathion and DDT when constitutively activated, through regulation of CYP6 genes and the glutathione transferase GSTD family [11, 12]. The cnc-Maf-S pathway has also been implicated in the regulation of the CYP families in the greenfly, Aphis gossypii [13] and beetles Tribolium castaneum and Leptinotarsa decemlineata $[14,15]$.

Maf-S is a small nuclear-located transcription factor that binds to antioxidant response elements (ARE) in the genome that control many genes involved in xenobiotic defence. Activation of transcription is regulated through heterodimerisation with a normally cytoplasmic protein, cnc. The formation of Maf-S-cnc complexes is in turn controlled by an actin binding ubiquitin ligase, Keap1. In the absence of electrophiles and reactive oxygen species, Keap 1 binds to cytoplasmic cnc targeting it for proteolysis [16]. Under conditions of stress, the interaction with Keap1 is disrupted, $c n c$ translocates to the nucleus, binds to Maf-S and activates ARE (Fig. 1). Genes putatively encoding the three components of this regulatory complex were identified in the genome of Anopheles gambiae, the major African malaria vector, by homology searches and examined for the presence of the functional domains: Maf-S is encoded by AGAP01045, cnc by AGAP005300 and Keap 1 by AGAP003645. Further information on each of these genes is provided in Additional file 1.

In this study, we show that Maf-S expression correlates with expression of multiple insecticide resistance candidates in the major malaria vector Anopheles

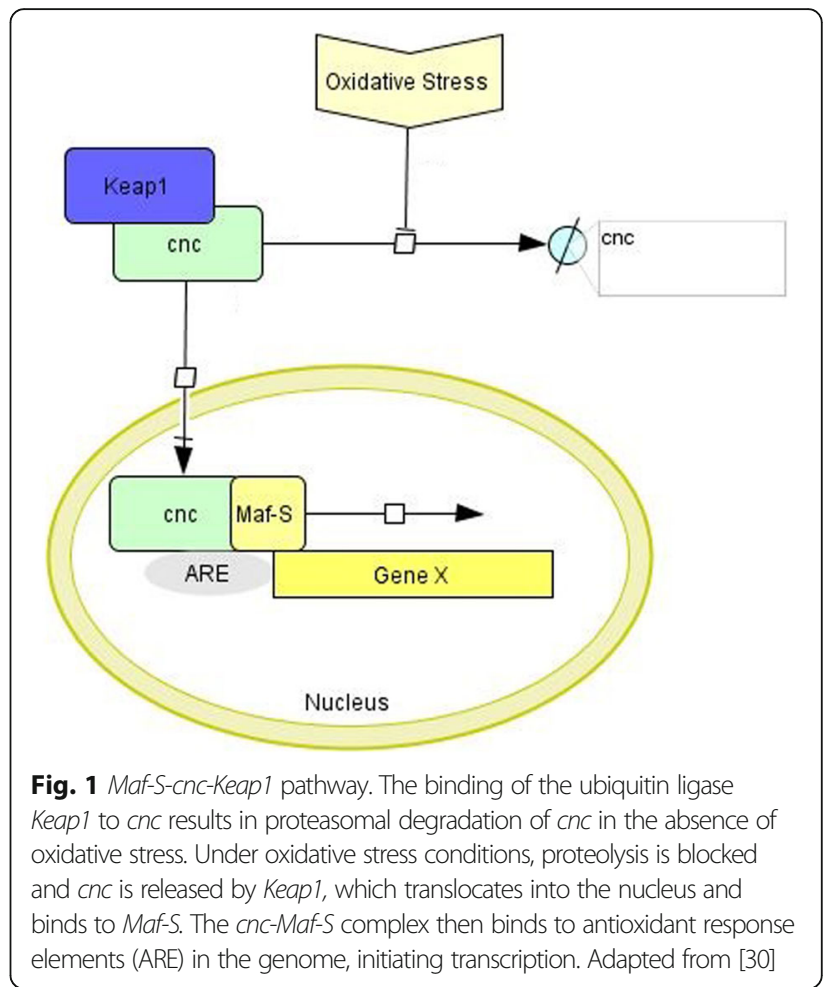

gambiae, suggestive of a regulatory role for this transcription factor in insecticide resistance. This was confirmed by RNAi mediated gene knockdown which resulted in an increase in the mosquitoes' susceptibility to pyrethroid and DDT insecticides but a decrease in their susceptibility to the organophosphate malathion. We hypothesise that this dichotomous response is mediated by one or more P450s, regulated by Maf-S, which are capable of both detoxifying and activating different insecticide classes.

\section{Results \\ Mining of transcriptomes from pyrethroid resistant populations to identify genes regulated by Maf-S}

Anopheles gambiae is a species complex of eight different species with three species, Anopheles gambiae s.s., Anopheles coluzzii and Anopheles arabiensis being the most important in terms of malaria transmission [17]. Pyrethroid resistance is now widespread in all three of these species [2] and previous studies to identify the molecular basis of this resistance have generated extensive datasets comparing expression of the entire transcriptome in insecticide susceptible or resistant populations (e.g [18-22]). These data sets have been mined individually to identify candidate effector genes responsible for the resistance phenotype (see references in Additional file 2: Table S1), several of which have been functionally validated [23-26]. In this study, we used these datasets to identify genes that were co-regulated 
with the Maf-S transcription factor. As variable study designs have been employed in the microarray experiments, we filtered the datasets to only consider experiments where gene expression was compared between a field collected pyrethroid resistant population and a standard susceptible population. A total of 27 data sets meeting the above criteria were identified (Additional file 2: Table S1).

The 27 microarray data sets were analysed using limma [27] to identify transcripts whose expression was strongly correlated with $M a f-S$ and co-factors, through use of correlation networks. Fourteen transcripts were strongly correlated (>0.8) with Maf-S expression (Additional file 3: Figure S2 and Additional file 4: Table S2) including two cytochrome P450s (CYP6M2 and CYP4H17) and two glutathione transferases (GSTD1 and GSTD3).

Expression of $c n c$ is correlated with 254 transcripts; GO term enrichments of these transcripts demonstrated roles in signalling, development and regulation and transcriptional cofactor activity, consistent with a role in transcriptional activation $(p \leq 0.05)$ (Additional file 4: Table S2). Keap1 had just three co-correlated transcripts, including TPX4, a juvenile hormone inducible protein and a GTPase (Additional file 4: Table S2). There was no overlap between the genes co-regulated with Maf$S$, cnc or Keap1 in the insecticide resistance microarray data sets (Additional file 4: Table S2) nor is there any evidence of co-regulation of these three transcripts in the larger data set of microarray experiments available on VectorBase [28]

\section{Maf-S knockdown}

To test whether Maf-S regulates expression of the pyrethroid resistance-associated transcripts, Maf-S levels were reduced via RNAi and then expression of transcripts encoding detoxification enzymes present in the correlation networks were quantified by qRT-PCR. Mosquitoes were first injected with two separate dsRNAs for $M a f-S$ and with $d s G F P$ used as a control. The degree of knockdown was determined by qPCR comparing Maf$S$ levels in GFP injected mosquitoes with either of the two Maf-S dsRNAs. The dsRNA with the most efficient knockdown of Maf-S, Maf-S (2), was used in all further experiments (ratio of Maf-S (1): 0.56 and Maf-S (2); 0.26 compared to GFP-injected controls) (Additional file 5: Figure S3).

Next, the expression of eight transcripts, five identified via the correlation network and three inferred from previous publications [11], were compared in mosquitoes injected with dsRNA against Maf-S compared to GFPinjected and uninjected controls. The eight genes comprised (i) four detoxification predicted by our correlation network to be co-regulated with Maf-S in An. gambiae
(GSTD1 (AGAP004164), GSTD3 (AGAP004382), CYP6M2 (AGAP008212) and CYP4H17 (AGAP008358)), (ii) an ABC transporter $A B C A 3$ (AGAP007504) also identified via the correlation network, (iii) orthologues of three genes shown to be regulated by the Maf-S-cnc-Keap1 pathway in D. melanogaster (two orthologs of the juvenile hormone epoxide hydrolases (Jheh: AGAP008684 and AGAP008685) and a glycine N-methyl transferase (gnmt, AGAP002198)) [11]. Suppressing expression of Maf-S resulted in reduced expression of six of the eight transcripts tested, two significantly (GSTD1 and Jheh1). Three of the four detoxification genes, CYP6M2, GSTD1 and GSTD3, were reduced as predicted by the correlation network whereas CYP4H17 showed elevated expression when compared to the GFP injected controls (Fig. 2). The correlation networks were not predictive of expression of the $\mathrm{ABC}$ transporter $A B C A 3$, which showed no clear change in expression after knock-down. All three of the transcripts selected from the genes regulated by Maf-S in Drosophila showed reduced expression in Maf-S knocked down groups suggesting that these genes are also regulated by the Maf-S pathway in mosquitoes (Fig. 2).

The up- and down-stream sequences from the annotated gene sequences were examined to identify potential motifs that may be involved in Maf-S transcription. The antioxidant response element (ARE) motif was first described in the mammalian system with a consensus motif 5' -TMAnnRTGAYnnGCRwwww-3' [29] and later identified downstream of GSTD1 in Drosophila in the

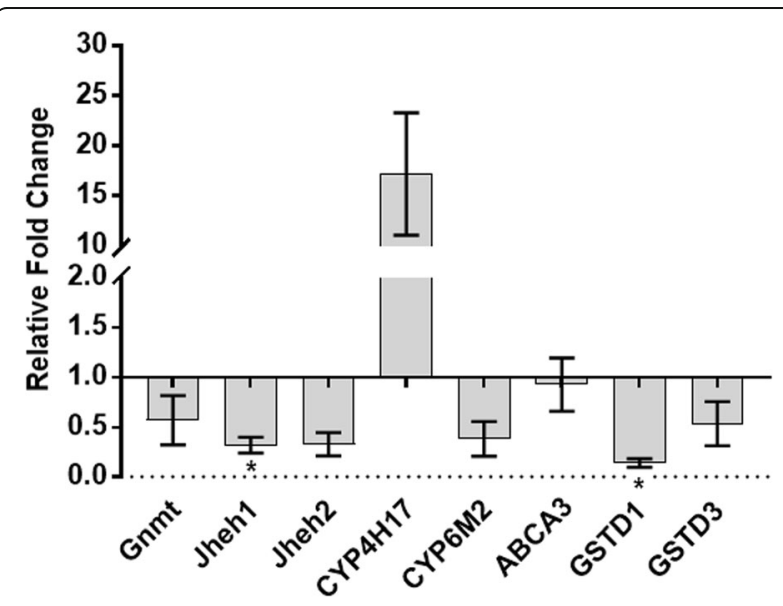

Fig. 2 Gene expression in Maf-S knockdowns relative to GFP injected controls. qPCR of three transcripts regulated by the cnc-Maf-S pathway in Drosophila (Gnmt, Jheh1, Jheh2) and five selected from the Maf-S co correlated genes in Anopheles (CYP4H17, GSTD3, ABCA3, CYP6M2, GSTD1) was performed on Maf-S knockdown CDNA. Data are shown normalised against expression in GFP-injected controls. Significance ( $p \leq 0.05)$, as determined by a Welch's t-test, is indicated by an *. Each data point represents the mean of three biological replicates each comprising cDNA from 7 to 10 females, $72 \mathrm{~h}$ post injection (actual age of mosquitoes $=7-8$ days) 
same form [30]; in both cases the ARE was shown to be the binding site of $c n c-M a f-S$. The presence of the Drosophila ARE motif, from JASPAR CORE Insect [31] (Additional file 6: Figure S4), was determined in 2000 base pairs upstream and downstream from each of the Maf-S co-correlated transcripts, using a motif searching package on $\mathrm{R}$ [32]. Twelve of the 14 transcripts identified by Maf-S correlation network analysis contained the ARE motif, including Maf-S itself, with 9 containing either an upstream or downstream ARE motif and a further two, GSTD3 and AGAP006662, containing both up and downstream ARE motifs (Additional file 6: Figure S4). ARE binding sites were not detected in $A B C A 3$ or $C Y P 4 H 17$, which is consistent with the observation that expression of these genes were not suppressed following knock-down of Maf-S (Fig. 2) but were detected in the flanking regions of the three Drosophila orthologs which we suppressed by Maf-S knockdown. Interestingly, $c n c$, Keap1 and Maf-S each contain up- and downstream occurrences of the ARE motif, which may be indicative of an auto-regulatory loop, similar to that described in mammalian systems and in Drosophila [30, 33, 34].

Having confirmed that Maf-S regulates expression of key detoxification genes in An. gambiae, we sought to obtain a more comprehensive picture of the genes controlled by this transcription factor by microarray analysis. RNA was extracted from the Tiassalé strain of An gambiae [35], $72 \mathrm{~h}$ post injection of $M a f-S(2)$ or GFP dsRNA and competitively hybridised to an $A n$. gambiae microarray [23]. A total of 3401 transcripts were significantly differentially expressed ( $p \leq 0.05$ ), of these 1703 were down-regulated in the Maf-S silenced group and 1698 were up regulated (Additional file 7: Table S3 and Additional file 8: Figure S5). The up-regulated transcript list is highly enriched in ion transport, ATP and purine processes and ligand channel activity whereas the down-regulated transcript list shows enrichment in key terms such as RNA binding, ribonucleoprotein and cytosol (Additional file 9: Table S4). Whilst it is recognised that the differentially expressed transcripts in the microarray experiment may include genes not directly regulated by $M a f-S$, it was encouraging that three of the six genes (GSTD1, CYP6M2, Gnmt) previously shown to be regulated by $M a f-S$ by qPCR were also significantly down regulated in the Maf-S silenced mosquitoes in the microarray dataset.

The differentially expressed genes included 64 transcripts from detoxification families commonly associated with insecticide resistance (glutathione transferases (GSTs), cytochrome P450s, carboxylesterases (COEs), UDP-glucuronosyltransferases (UGTs) and ABC transporters), 30 in the up regulated list and 34 in the down regulated list (Additional file 10: Table S5). The genes, down-regulated after Maf-S silencing, included 17 P450s, two ABC transporters, eight GSTs, four UGTs and three COEs (Additional file 10: Table S5 and Additional file 8: Figure S5). In addition to CYP6M2 the down-regulated P450s included CYP6Z2, CYP6Z3, CYP6P4, CYP4G16 and CYP4G17 and GSTD1, all of which have been associated with insecticide resistance in An. gambiae [36]. Two further cytochrome P450s linked to ecdysone biosynthesis were also down - regulated by Maf-S silencing: CYP302A1 and CYP314A1 [37]. Detoxification genes with higher numbers of transcripts following Maf-S silencing included five ABCs, six COEs, 15 cytochrome P450s, two GSTs and two UGTs. Of these only CYP9K1 is a strong candidate for conferring insecticide resistance in An. gambiae [38].

\section{Maf-S regulates the response to insecticides in anopheles} In Drosophila the Maf-S-cnc-Keap1 pathway has been shown to play a role in resistance to both malathion and DDT [12]. In order to evaluate the role of this pathway in insecticide resistance in An. gambiae, Maf-S knockdowns were again produced by RNAi experiments in the Tiassalé strain. This strain, originally colonised from rice fields in southern Cote d'Ivoire [35] is resistant to DDT, carbamates and pyrethroids but susceptible to the organophosphate malathion according to World Health Organisation definitions [39]. Maf-S knocked down Tiassalé mosquitoes were exposed to insecticides $72 \mathrm{~h}$ after RNAi injection and mortality recorded a further $24 \mathrm{~h}$ later. The discriminating doses set by WHO were used for all insecticides and the standard $60 \mathrm{~min}$ exposure used for the pyrethroids, bendiocarb and DDT but, as the uninjected controls were all killed by $60 \mathrm{~min}$ exposure to the discriminating dose of malathion, shorter exposure times of $5 \mathrm{~min}$ were used to detect the effect of $M a f-S$ silencing on insecticide susceptibility.

Silencing $M a f-S$ significantly increased mortality (i.e. reduced the resistance) of the Tiassale strain to DDT ( $\mathrm{p}=<0.01)$, and the pyrethroids deltamethrin and permethrin ( $p=0.036$ and $p=0.015$, respectively) but had no impact on bendiocarb induced mortality (Fig. 3). In contrast, silencing Maf-S decreased the susceptibility of the mosquitoes to malathion (increased their resistance) $(\mathrm{p}=<0.01)$. Unexpectedly, mosquitoes injected with dsGFP also showed lower mortality after malathion exposure than the uninjected controls, nevertheless, there was a significantly reduced mortality in the $M a f-S$ versus GFP injected groups $(p=0.0054)$. Malathion is a proinsecticide, which is activated to the insecticidal oxon form by P450s. The observation that supressing the $c n c$ Maf-S pathway increases pyrethroid toxicity but decreases malathion toxicity in vivo suggests that constitutive elevation of this pathway could result in resistance to some insecticide classes whilst simultaneously increasing the susceptibility to other insecticide classes. 


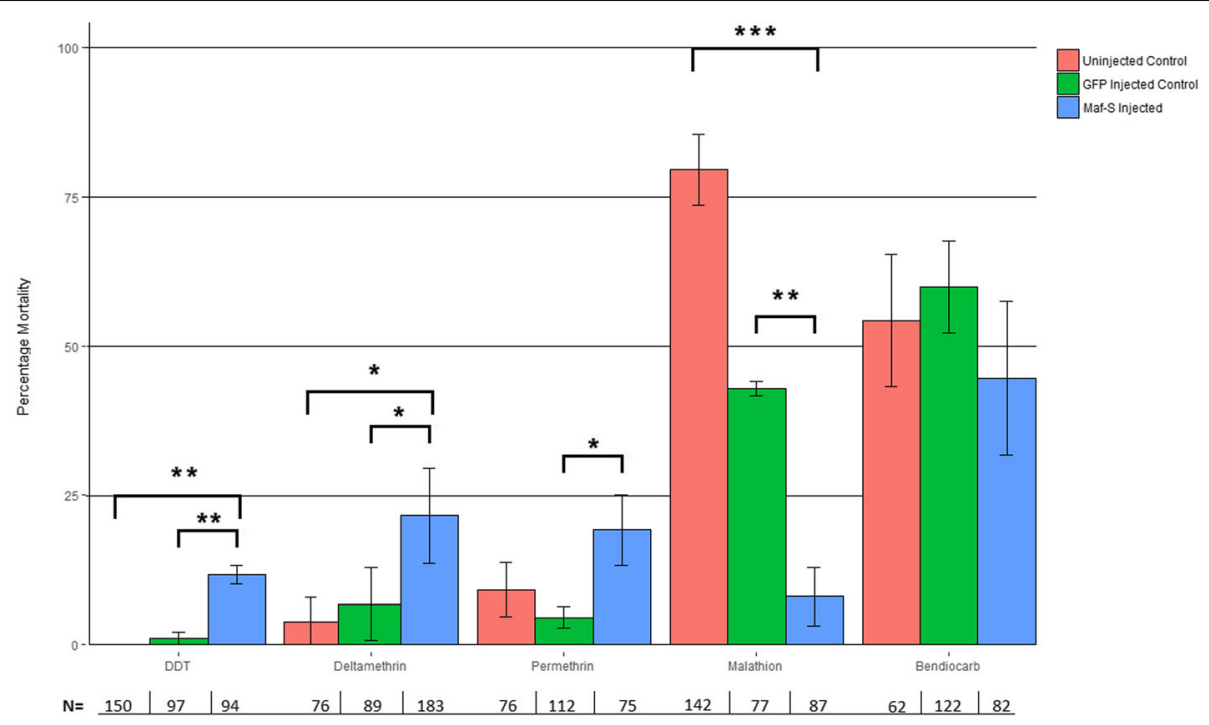

Fig. 3 Insecticide bioassays on Maf-S silenced adults. An. gambiae sl female mosquitoes were injected with Maf-S or GFP dsRNA, and then exposed to insecticides using the WHO tube assay. Exposure times were 60 min to papers coated with $4 \%$ DDT, $0.75 \%$ permethrin, $0.05 \%$ deltamethrin or $0.1 \%$ bendiocarb; and 5-min to 5\% malathion papers. Significance is represented by: ${ }^{*} p \leq 0.05,{ }^{* *} p \leq 0.01$ and ${ }^{* * *} p \leq 0.001$ displayed above each bar, as calculated by ANOVA with a Tukey post hoc test. Error bars represent standard error. Number of mosquitoes per test for each insecticide/condition are represented by numbers below the $x$-axis. Each WHO test had 20-25 female mosquitoes per tube

This 'negative cross resistance' has major operational implications for malaria control, as discussed below.

\section{Discussion}

Significant progress has been made in recent years in identifying the metabolic enzymes responsible for elevated detoxification in insecticide resistant pest species [40]. However, the central question of the mechanisms underpinning increased gene expression are poorly understood. Copy number variation has been implicated in elevated P450 levels in some insecticide resistant populations but in most cases, even where gene duplication does occur, transcriptional regulation is thought to be involved [41, 42]. The $c n c-M a f-S$ pathway has recently been shown to be conserved and constitutively activated in DDT- and malathion-resistant Drosophila melanogaster [11, 12], deltamethrin-resistant red flour beetles [14] and imidacloprid-resistant Colorado potato beetles [15]. The initial objective of this study was to determine whether the same pathway was also active in pyrethroid resistant populations of malaria vectors. To do this we took advantage of previously generated datasets comparing transcriptomes of pyrethroid resistant and susceptible An. gambiae populations and used a bioinformatics approach to identify genes that appeared to be co-regulated with the three components of the complex. We identified a large number of genes whose expression was correlated with $c n c$ transcript levels, several of which may be related to the multiple roles of $c n c$ in homeostasis, development, metabolism and aging [43]. A much smaller number of genes (14) were identified in the correlation networks of $M a f-S$ and nearly half of these belonged to four gene families typically implicated in insecticide metabolism and transport.

Next, we used RNAi to knockdown expression of Maf-S and confirmed that transcripts of a subset of these co-regulated genes were also depleted in the Maf-S knockdowns. Included amongst these genes were CYP6M2, a proven pyrethroid metaboliser [26] and GSTD1, which catalyses the dehydrochlorination of DDT [25]. Subsequent microarray experiments also identified further detoxification gene transcripts suppressed by Maf-S silencing including, CYP6Z2, CYP6Z3, CYP4G16, CYP4G17, and CYP6P4 all previously linked to pyrethroid and/or DDT resistance [18-22, 36]. Although a similar number of detoxification genes were found to have elevated expression in the Maf-S silenced mosquitoes, this subset of genes does not contain any with known affinity for binding and/or metabolising insecticides and expression of only one of these, CYP9K1, has been associated with insecticide resistance [38]. Although the ARE motif is present downstream of CYP6P3 a pyrethroid metaboliser associated with pyrethroid resistance in multiple populations [24], this gene was not significantly differentially expressed in the microarray data set after Maf-S knockdown; this may indicate that CYP6P3 is regulated independently of this pathway although it should also be noted that the microarray experiments did not detect all of the genes we confirmed to be regulated by $M a f-S$ by qPCR. 
Enrichment of Maf-S expression in the midgut and malpighian tubules $[36,44]$ (tissues implicated in detoxification in insects $[37,45]$ ), and the importance of this transcription factor in controlling expression of genes encoding known insecticide metabolising enzymes, were supportive of a role in insecticide resistance [36]. This was confirmed by insecticide bioassays which showed that supressing expression of Maf-S resulted in an increase in susceptibility to the pyrethroids deltamethrin and permethrin, and the organochlorine DDT, in the Tiassalé strain which is normally highly resistant strain to these insecticide classes $[35,46]$. We note that susceptibility was not fully restored after Maf-S silencing; this was not unexpected as the Tiassalé strain is known to contain additional resistance mechanisms, such as target site mutations, that are not regulated by Maf-S. Furthermore, injection of dsRNA did not fully silence expression of this gene, and this coupled with the unknown turnover rates for detoxification proteins, may have resulted in continued expression of Maf-S regulated genes, all be it at a reduced level.

Previous microarray studies have identified a subset of P450 genes that are elevated in the Tiassalé resistant strain compared to susceptible populations [20]; these include CYP6M2, CYP6P3, CYP6P4, CYP6Z2 and CYP6Z3. We show that all of these P450s, with the exception of $C Y P 6 P 3$, are regulated by Maf-S; furthermore several of these P450s have been shown to metabolise pyrethroids and/or DDT via in vitro characterisation of recombinant enzymes (26 and our unpublished data). Our data therefore suggests that depletion of Maf-S reduces levels of key pyrethroid and DDT detoxifying enzymes and increases the susceptibility of the resistant strain to these chemicals. Other detoxification genes regulated by $M a f-S$, but with as yet uncharacterised roles in insecticide metabolism, are also over expressed in the Tiassalé resistant strain (see Additional file 10: Table S5) and these warrant further investigation.

The cnc-Maf-S pathway in invertebrates has multiple functions in addition to its role in mediating response to oxidative stress and xenobiotics. For example the cncMaf-S heterodimer is involved in Drosophila development [16] and control of energy metabolism [47, 48]. Furthermore, in Drosophila, cnc-Maf-S plays a part in ecdysone biosynthesis [49]. Ecdysteroids determine moulting timing, and their precursors are modified by a subset of cytochrome P450s known as the Halloween genes: Phantom (CYP306A1), disembodied (CYP302A1), shadow (CYP315A1) and shade (CYP314A1). Of these genes, $C Y P 302 A 1$ and $C Y P 314 A 1$ were both down regulated after Maf-S silencing in this study. Furthermore, two juvenile hormone esterase hydrolases which catalyse the enzymatic degradation of juvenile hormone [50] are also regulated by Maf-S in Anopheles and Drosophila [11,12, this study] supporting a role for this pathway in metamorphosis.

\section{An unexpected impact of disruption of the Maf-S path- way on malathion resistance.}

Whilst attenuating the Maf-S pathway decreased the mosquitoes' ability to withstand DDT and pyrethroid exposure, it had a dramatic opposing effect on the response to malathion exposure. The Tiassalé strain of mosquitoes is highly susceptible to malathion (exposure to the discriminating dose for $60 \mathrm{~min}$ resulted in 100\% mortality). Surprisingly however, when mosquitoes were exposed for $5 \mathrm{~min},<10 \%$ in the Maf-S silenced population were killed compared to $80 \%$ of the uninjected control and $43 \%$ of the GFP control. Malathion is a proinsecticide which is activated to the much more toxic oxon form in the insect; this oxidative desulfuration reaction is catalysed by P450s [51]. As described above, CYP6M2, along with members of the CYP6P and CYP6Z family are the most highly upregulated P450s in the insecticide resistant Tiassalé strain [52]. CYP6M2, has a high affinity for malathion and the primary metabolite after incubating malathion with recombinant CYP6M2 has a molecular mass of 315 , consistent with the activated form malaoxon [53]. Thus, the decreased malathion induced toxicity observed could be explained by a reduction in CYP6M2 levels in the Maf-S knockdowns, as confirmed in both the array and qPCR results, reducing the rate of activation of malathion to malaoxon and reducing the toxic effect of the insecticide in these knockdown mosquitoes.

Interestingly, the opposite phenotype was observed in D. melanogaster. Here activating the Maf-S pathway, by depletion of the repressor Keap1, resulted in increased resistance to malathion [12] suggesting that the enzymes responsible for malathion detoxification, and not activation, are regulated by Maf-S in this species. Clear 1:1 orthology is rare in the Anopheles and Drosophila P450 families [54] and as far as we are aware, the P450s activating malathion in Drosophila have not been identified and thus it is not yet possible to validate this prediction. However, the finding that perturbing a single transcription factor in Anopheles can increase susceptibility to one insecticide class whilst simultaneously reducing the susceptibility to a chemically unrelated class provides important insights into metabolic resistance to insecticides which may have operational implications for resistance management.

Experimental hut studies from Côte d'Ivoire found malathion to be highly effective against the pyrethroid resistant Tiassalé population and it has been postulated that 'negative cross resistance' may be enhancing the 
efficacy of organophosphates in this setting [55]. Negative cross resistance between pyrethroids and proinsecticides, mediated by elevated cytochrome P450 activity in the insects, has been demonstrated in agricultural pests [56] but has so far remained a largely untested theoretical hypothesis in mosquitoes. Here we provide evidence for its existence, and identify both an effector enzyme and regulatory pathway. CYP6M2 is upregulated in the Tiassale population in Côte d'Ivoire [52] and thus this $\mathrm{P} 450$ (and/or possibly others regulated by the $c n c-M a f-S$ ) pathway could both enhance the efficacy of pyrethroids whilst also increasing the potency of malathion (and potentially other pro-insecticides).

\section{Conclusion}

This study provides novel insights into the transcriptional regulation of insecticide resistance in the malaria vector, $A n$. gambiae. We demonstrate that the transcription factor $M a f-S$ controls expression of multiple detoxification-related transcripts, including the proven pyrethroid and DDT metabolisers, CYP6M2 and GSTD1. Secondly we show that silencing expression of Maf-S leads to increased susceptibility to these insecticides. Finally, we provide evidence that the $M a f-S$ pathway regulates one or more P450s responsible for the negative cross-resistance between pyrethroids and organophosphates. Malaria endemic countries are facing a public health crisis as resistance erodes the efficacy of the limited chemicals available to target the mosquito vectors [57]. The results from this study highlight the value of understanding the molecular basis of resistance and offer hope that this information can be used to introduce effective strategies to manage resistance.

\section{Methods}

\section{Microarray analysis}

Microarray datasets listed in (Additional file 2: Table $\mathrm{S} 1$ ), and associated metadata were provided by members of the Department of Vector Biology at LSTM and are publicly accessible in ArrayExpress (Accession numbers listed in Additional file 2), VectorBase or through the respective publications. All microarray datasets were analysed using the base limma $R$ package [58], applying linear models to correct and normalise data, inferring differential transcript expression. Data was normalised using affycoretools [59]. Both within (loess) and between (aquantile) arrays, in addition to background correction (mle) were performed. Dye swap correction and design matrices were used where necessary. False discovery rate testing was used for multiple test correction. All other parameters were kept as default. All information for use can be found on Bioconductor (https://bioconductor.org/packages/release/bioc/html/limma.html).
Probability of a transcript being differentially regulated in $\mathrm{n}$ arrays by chance

Each of the microarray datasets listed in Additional file: Table S1 were used to calculate the probabilities of success (significance) and failure (non-significance) of a given transcript. The probability of success was calculated using the average number of all significant transcripts as a proportion of the overall number of transcripts on the array. The probability of success, over all arrays, was 0.4692 and that of failure, 0.5308 . These data were used to calculate whether each transcript in the Maf-S-cnc pathway was significant in more data sets than expected by chance.

\section{Enrichment tests}

Enrichments of transcript lists for both correlation networks and microarray data were performed using the DAVID functional annotation tool (https://david.ncifcrf.gov/summary.jsp) [60] for transcript lists that were longer than 100 transcripts. Smaller transcript lists were assessed for specific enrichments using a hypergeometric test in R. In both cases, significance was determined as $p \leq 0.05$.

\section{Correlation networks}

Correlation networks were produced using a correlation matrix with a Euclidean distance metric produced in $\mathrm{R}$, with fold changes of all transcripts from all 27 available microarrays (Additional file 2: Table S1). To identify only genes with a strong correlation or anti-correlation, only those transcripts with a correlation of \pm 0.8 were used. These data were extracted from the correlation matrix along with associated $\log _{2}$ fold change for each of the separate microarray experiments and viewed in Cytoscape [61] as a biological network.

\section{Mosquito rearing conditions}

The An. gambiae s.l used in these experiments were from the Tiassalé strain originally from Côte D'Ivoire but maintained under pyrethroid selection pressure in the insectaries at the Liverpool School of Tropical Medicine since 2013. This strain is resistant to pyrethroids and DDT but susceptible to malathion [35, 52] (Fig. 3). Mosquitoes were reared under standard insectary conditions at $27{ }^{\circ} \mathrm{C}$ and $70-80 \%$ humidity under a $12: 12 \mathrm{~h}$ photoperiod.

\section{RNAi}

RNAi constructs were produced for $M a f-S$ in the form of dsRNA for microinjections (Additional file 11: Table S6). PCR was performed using Phusion ${ }^{\bullet}$ High-Fidelity DNA Polymerase (Thermo Scientific) following manufacturer's instructions and primer sets with a T7 docking sequence at the $5^{\prime}$ end of both the sense and antisense primers (Additional file 11: Table S6). Primers were designed to 
produce an asymmetric product with a length of 300$600 \mathrm{bp}$, a GC content of $20-50 \%$ and no more than three consecutive equivalent nucleotides. PCR was performed with the following cycle: three minutes $98^{\circ} \mathrm{C}, 35$ cycles of seven seconds at $98^{\circ} \mathrm{C}$ and $10 \mathrm{~s}$ at $72^{\circ} \mathrm{C}$, with a final hold at $72^{\circ} \mathrm{C}$ for seven minutes. PCR products were resolved on $1 \%$ agarose gels for $45 \mathrm{~min}$ and the correct length amplifications identified. The PCR products were purified using a Qiagen QIAquick PCR Purification kit following manufacturer's instructions. dsRNA was synthesised using a Megascript $^{\circ} \mathrm{T} 7$ Transcription (Ambion) kit, with a $16 \mathrm{~h} 37^{\circ} \mathrm{C}$ incubation, following manufacturer's instructions. The dsRNA was then cleaned using a MegaClear ${ }^{\circ}$ Transcription Clear Up (Ambion) kit, with DEPC water, twice heated at $65^{\circ} \mathrm{C}$ for $10 \mathrm{~min}$, to elute the sample. The resultant dsRNA product was analysed using a nanodrop spectrometer (Nanodrop Technologies, UK) and subsequently concentrated to $3 \mu \mathrm{g} / \mu \mathrm{l}$ using a vacuum centrifuge at $35^{\circ} \mathrm{c}$. Injections were then carried out using a nanoinjector with $69 \mathrm{nl}$ of product injected directly into the thorax, between the cuticle plates of the abdomen, underneath the wing. Injections were carried out on 100, three-to-five day old, presumed mated, non-blood fed females, which were immobilised on a $\mathrm{CO}_{2}$ block. As a control, nonendogenous GFP dsRNA was injected at the same amount and concentration [62].

\section{Microarrays}

A whole-genome microarray approach was used to determine the effect of Maf-S knockdown on transcriptional profiles. The transcriptional profiles of Maf-S knockdowns were compared against a GFP injected control. RNA was extracted from three biological replicates for each of Maf$S(2)$ injected and GFP injected controls. Mosquitoes were collected $72 \mathrm{~h}$ post injection, between the hours of $8 \mathrm{am}$ and $2 \mathrm{pm}$. Each replicate was added to extraction buffer from the PicoPure RNA extraction kit, heated for $30 \mathrm{~min}$ at $42^{\circ} \mathrm{C}$ and frozen at $-80^{\circ} \mathrm{C}$ as per manufacturer's instructions. Each biological replicate for each treatment consisted of RNA, extracted using PicoPure RNA Isolation kit (Arcturus), from 7 to 12 three-five day old non-blood fed, presumed mated females. The quantity and quality of the RNA was assessed using a nanodrop spectrophotometer (Nanodrop Technologies UK) and Bioanalyser (Agilent) respectively. $100 \mathrm{ng}$ of RNA was amplified and labelled with $\mathrm{Cy} 3$ and $\mathrm{Cy} 5$, using the Two colour low input Quick Amp labelling kit (Agilent) following the manufacturer's instructions. Samples were then purified (Qiagen) with the cRNA yield and quality assessed using the nano-drop and Bioanalyser respectively. RNA from each Maf-S injection replicate was competitively hybridised with the GFP injected control replicates. Dye swaps were performed on each of the technical replicates for each array, to correct for dye bias. Labelled cRNAs were hybridised to the whole genome 8x15k Anopheles gambiae array (ArrayExpress accession number A-MEXP-2211). Microarray hybridisation, washing and scanning were performed according to previously described protocols [23]. The experiment was submitted to ArrayExpress, accession E-MTAB-4042.

\section{qPCR}

qPCR was performed on total RNA extracted from post knockdown Tiassalé cDNA 3 days after injection using the following transcripts: AGAP008212-RA, AGAP004382-RA, AGAP008358-RA, AGAP002198-RA, AGAP008685-RA, AGAP008684-RA, AGAP004164-RA and AGAP007504RA. RNA $(4 \mu \mathrm{g})$ from each biological replicate $(n=3$ per treatment group) was reverse transcribed using Oligo dT (Invitrogen) and Superscript III (Invitrogen) according to manufacturer's instructions, DNase I (Qiagen) was applied to the column for $15 \mathrm{~min}$ at room temperature to remove any gDNA contamination. Quantitative real-time PCR was performed using SYBR Green Supermix III (Applied Biosystems) using an MX3005 and the associated MxPro software (Agilent). Primer Blast (NCBI) [63] was used to design primer pairs (Additional file 11: Table S6). Primers were designed to span an exon junction, where possible. Each $20 \mu \mathrm{l}$ reaction contained $10 \mu \mathrm{l}$ SYBR Green Supermix, $0.3 \mu \mathrm{M}$ of each primer and $1 \mu \mathrm{l}$ of 1:10 diluted cDNA. Standard curves were produced using whole Tiassalé cDNA, in 1, 1:5, 1:25, 1:125 dilutions. qPCR was performed with the following conditions: $3 \mathrm{~min}$ at $95^{\circ} \mathrm{C}$, with 40 cycles of $10 \mathrm{~s}$ at $95^{\circ} \mathrm{C}$ and $10 \mathrm{~s}$ at $60^{\circ} \mathrm{C}$. All amplification efficiencies of designed primers were within acceptable range (90$120 \%$ ), following MIQE guidelines [64]. Data was analysed using the $\Delta \Delta$ ct method [65], with GFP injected control as the comparator. All data were normalised against two housekeeping genes: S7 and EF (Additional file 11: Table S6). Welch's t-tests were performed on the $\Delta$ ct values, with significance of $p \leq 0.05$

\section{Bioassays}

72-h post injection, a minimum of 75 female mosquitoes were assayed using WHO bioassay tube test kits [39] (minimum of 3 biological replicates of 25 mosquitoes). These assays were used under standard conditions for one-hour exposures to $0.05 \%$ deltamethrin, $0.75 \%$ permethrin, $4 \% \mathrm{DDT}, 0.01 \%$ bendiocarb and 5 min exposures to $5 \%$ malathion impregnated papers. Each exposure had a corresponding untreated control of 25 female mosquitoes. Post-exposure, mosquitoes were left in a control tube, under insectary conditions for $24 \mathrm{~h}$ and mortality recorded. Significance tests were carried out using ANOVA with a Tukey post hoc test. Homogeneity of variance and normality of data were checked using a Bartlett test and a Shapiro Wilk test respectively; all data was transformed using an arcsine transformation. 


\section{Additional files}

Additional file 1: Figure S1. Details of the genes encoding Maf-S cnc and Keap1 in An gambiae. (DOCX 37 kb)

Additional file 2: Table S1. Information on microarray data sets from insecticide resistant populations. Population name represents the area from which the strain was sampled in addition to insecticide exposure, where relevant. Country of origin, species, susceptible reference strain and paper reference are also given. (XLSX $10 \mathrm{~kb}$ )

Additional file 3: Figure S2. Maf-S co-correlated transcripts. $\log _{2}$ fold change (y) of Maf-S and co-correlated transcripts across the 27 microarray studies ( $x$ axis labels) described in Additional file 2: Table S1. (PNG 37 kb)

Additional file 4: Table S2. Co-correlated transcripts for each of $c n c_{\text {, }}$ Maf-S and Keap1. Gene ID and associated description for all co-correlated transcripts $( \pm 0.8)$ for each gene in the Maf-S-cnc-Keap1 pathway. Correlation was computed via a correlation matrix in $\mathrm{R}$ using the microarray datasets in Additional file 2: Table S1. (XLSX 19 kb)

Additional file 5: Figure S3. dsRNA knockdown levels. (a) Relative transcript levels in dsRNA Maf-S injected mosquitoes compared to GFP-injected and uninjected controls following injection of two alternative Maf-S dsRNA constructs, Maf-S(1) and Maf-S(2). (b) Relative transcript levels for each splice variant (Maf-S-RA: AGAP010405-RA (RA) and Maf-S-RB: AGAP010405-RB (RB)) following injection of the Maf-S(2) dsRNA construct. For details of primers see Additional file 11: Table S6. Error bars represent the standard error of the mean. (JPEG $37 \mathrm{~kb}$ )

Additional file 6: Figure S4. Anti-oxidant response element motif and corresponding presence/absence in up- and down-stream regions of Maf-S co-correlated transcripts. (a) JASPAR Core Insect motif representing the antioxidant- or xenobiotic- response element. (b) Presence of the motif $2000 \mathrm{bp}$ up- and down-stream of Maf-S co-correlated transcripts, showing transcript $I D$, region of motif location and the representative motif. (PNG $78 \mathrm{~kb}$ )

Additional file 7: Table S3. Significant transcripts on Maf-S-RNA microarray data set. Transcript ID, $\log _{2}$ fold change, adjusted $p$-value and B-value as calculated by limma analysis for all significant transcripts on the arrays comparing Maf-S silenced to GFP-injected controls. (XLSX 198 kb)

Additional file 8: Figure S5. Volcano plot showing transcript levels in Maf-S knockdowns compared to GFP controls. Significantly differentially expressed probes are shown in black (adjusted $p \leq 0.05$ ), detoxification family members are shown in shapes indicated on the key $(A B C=A B C$ transporter, $\mathrm{COE}=$ carboxylesterase, $\mathrm{CYP}=$ cytochrome $\mathrm{p} 450, \mathrm{GST}=$ glutathione-S-transferase and UGT = (UDP-glucuronosyltransferase). Probes are labelled with associated transcript IDs/gene names where $p \leq 0.001$. Genes down-regulated in the Maf-S knockdowns probes are shown to the left and up-regulated probes to the right. (PNG $88 \mathrm{~kb}$ )

Additional file 9: Table S4. Significant GO terms for significantly up- and down-regulated transcripts. Significant GO terms for transcript lists significantly up- (bold) or down- (italic) regulated after Maf-S knockdown. GO terms, Benjamini adjusted $p$-values and transcript membership shown as calculated by DAVID [66]. (XLSX 10 kb)

Additional file 10: Table S5. Detoxification transcripts significantly differentially expressed in Maf-S microrarray. 64 transcripts belonging to Phase I, II or III detoxification family members (glutathione transferases, cytochrome P450s, UGTs and ABCs) that show significant differential expression after knockdown of Maf-S. In addition to fold change from the Maf-S knockdown arrays data from previously published experiments comparing gene expression between Tiassalé and a susceptible population, N'Gousso to indicate which of the genes regulated by Maf-S are differentially expressed in the Tiassalé populations and thus candidates for conferring resistance [20]. (XLSX $12 \mathrm{~kb}$ )

Additional file 11: Table S6. Primer Tables. RNAi primers used in the synthesis of dsRNA, each with a T7 promoter region (shown in lower case) and sequences of primers used in all qPCR validation. (XLSX $11 \mathrm{~kb}$ )

\section{Abbreviations}

ARE: Antioxidant Response Element; cDNA: Complementary DNA; COE: Carboxylesterase; cRNA: Complementary RNA; dsRNA: Double Stranded RNA; GFP: Green Fluorescent Protein; GO: Gene Ontology; GST: Glutathione-
S-Transferase; IRS: Indoor residual spraying; qPCR: Quantitative PCR; RNAi: RNA Interference; UGT: UDP-glucuronosyltransferas

\section{Acknowledgments}

Not Applicable.

\section{Author contributions}

$\mathrm{VI}$ and PP performed all experiments. $\mathrm{VI}$ and H.R designed the study. VI, SW and JM analysed all data. VI and HR drafted the manuscript. All authors read and approved the final manuscript.

\section{Funding}

The research leading to these results was supported by the European Union Seventh Framework Programme FP7 (2007-2013) under grant agreement no 265660 AvecNet.

\section{Availability of data and materials}

The dataset supporting the conclusions of this article is available in the ArrayExpress repository, E-MTAB-4042 (https://www.ebi.ac.uk/arrayexpress/experiments/E-MTAB-4042/).

Ethics approval and consent to participate

Not Applicable.

Consent for publication

Not Applicable.

Competing interests

The authors declare that they have no competing interests.

\section{Publisher's Note}

Springer Nature remains neutral with regard to jurisdictional claims in published maps and institutional affiliations.

\section{Author details}

${ }^{1}$ Liverpool School of Tropical Medicine, Pembroke Place, Liverpool, England, L35QA. 'Earlham Institute, Norwich Research Park Innovation Centre, Colney Lane, Norwich, EnglandNR4 $7 \mathrm{UH}$.

Received: 15 May 2017 Accepted: 24 August 2017

Published online: 30 August 2017

\section{References}

1. WHO. World Malaria Report. Geneva; 2015

2. Ranson $\mathrm{H}$, Lissenden $\mathrm{N}$. Insecticide resistance in African anopheles mosquitoes: a worsening situation that needs urgent action to maintain malaria control. Trends Parasitol Elsevier. 2016:32:187-96.

3. Ranson H, N'Guessan R, Lines J, Moiroux N, Nkuni Z, Corbel V. Pyrethroid resistance in African Anopheline mosquitoes: what are the implications for malaria control? Trends Parasitol. 2011;27:91-8

4. WHO. Global plan for insecticide resistance management in malaria vectors (GPIRM). 2012. http://www.who.int/malaria/publications/atoz/gpirm/en/. Accessed May 2012

5. Donnelly MJ, Isaacs AT, Weetman D. Identification, validation, and application of molecular diagnostics for insecticide resistance in malaria vectors. Trends Parasitol. Elsevier. 2015;32:197-206.

6. Wang S, Hankinson O. Functional involvement of the Brahma/SWI2-related gene 1 protein in cytochrome P4501A1 transcription mediated by the aryl hydrocarbon receptor complex. J. Biol. Chem. ASBMB. 2002;277:11821-7.

7. Gao J, Xie W. Pregnane X receptor and constitutive androstane receptor at the crossroads of drug metabolism and energy metabolism. Drug Metab Dispos ASPET. 2010;38:2091-5.

8. Shen G, Kong A. Nrf2 plays an important role in coordinated regulation of phase II drug metabolism enzymes and phase III drug transporters. Biopharm Drug Dispos Wiley Online Library. 2009:30:345-55.

9. Brown RP, McDonnell CM, Berenbaum MR, Schuler MA. Regulation of an insect cytochrome P450 monooxygenase gene (CYP6B1) by aryl hydrocarbon and xanthotoxin response cascades. Gene. 2005;358:39-52.

10. King-Jones K, Horner MA, Lam G, Thummel CS. The DHR96 nuclear receptor regulates xenobiotic responses in drosophila. Cell Metab. 2006;4:37-48. 
11. Misra JR, Horner MA, Lam G, Thummel CS. Transcriptional regulation of xenobiotic detoxification in drosophila. Genes Dev. 2011;25:1796-806.

12. Misra JR, Lam G, Thummel CS. Constitutive activation of the Nrf2/Keap1 pathway in insecticide-resistant strains of drosophila. Insect Biochem Mol Biol. 2013:43:1116-24.

13. Peng T, Pan Y, Gao X, Xi J, Zhang L, Yang C, et al. Cytochrome P450 CYP6DA2 regulated by cap ' $n$ 'collar isoform C ( $\mathrm{CncC}$ ) is associated with gossypol tolerance in Aphis Gossypii glover. Insect Mol Biol Wiley Online Library. 2016;25:450-9.

14. Kalsi M, Palli SR. Transcription factors, CncC and Maf, regulate expression of CYP6BQ genes responsible for deltamethrin resistance in Tribolium Castaneum. Insect Biochem Mol Biol Elsevier. 2015;65:47-56.

15. Kalsi M, Palli SR. Transcription factor cap $n$ collar $\mathrm{C}$ regulates multiple cytochrome P450 genes conferring adaptation to potato plant allelochemicals and resistance to imidacloprid in Leptinotarsa Decemlineata (say). Insect Biochem. Mol. Biol. Elsevier. 2017;83:1-12.

16. Veraksa A, McGinnis N, Li X, Mohler J, McGinnis W. Cap 'n'collar B cooperates with a small Maf subunit to specify pharyngeal development and suppress deformed homeotic function in the drosophila head. Development. 2000;127:4023-37.

17. Fontenille D, Simard F. Unravelling complexities in human malaria transmission dynamics in Africa through a comprehensive knowledge of vector populations. Comp Immunol Microbiol Infect Dis Elsevier. 2004;27:357-75.

18. Hemingway J, Vontas J, Poupardin R, Raman J, Lines J, Schwabe C, et al. Country-level operational implementation of the global plan for insecticide resistance management. Proc Natl Acad Sci. 2013;110:9397-402.

19. Jones CM, Haji KA, Khatib BO, Bagi J, Mcha J, Devine GJ, et al. The dynamics of pyrethroid resistance in anopheles arabiensis from Zanzibar and an assessment of the underlying genetic basis. Parasit Vectors. 2013;6:343.

20. Toé $\mathrm{KH}$, N'Falé $\mathrm{S}$, Dabiré RK, Ranson $\mathrm{H}$, Jones $\mathrm{CM}$. The recent escalation in strength of pyrethroid resistance in anopheles coluzzi in West Africa is linked to increased expression of multiple gene families. BMC Genomics. 2015;16:146.

21. Abdalla H, Wilding CS, Nardini L, Pignatelli P, Koekemoer LL, Ranson H, et al. Insecticide resistance in anopheles arabiensis in Sudan: temporal trends and underlying mechanisms. Parasit Vectors. 2014;7:213.

22. Mawejje HD, Wilding CS, Rippon EJ, Hughes A, Weetman D, Donnelly MJ. Insecticide resistance monitoring of field-collected Anopheles Gambiae sl populations from Jinja, eastern Uganda, identifies high levels of pyrethroid resistance. Med Vet Entomol Wiley Online Library. 2013;27:276-83.

23. Mitchell SN, Stevenson BJ, Müller P, Wilding CS, Egyir-Yawson A, Field SG, et al. Identification and validation of a gene causing cross-resistance between insecticide classes in Anopheles Gambiae from Ghana. Proc Natl Acad Sci. 2012:109:6147-52.

24. Müller P, Warr E, Stevenson BJ, Pignatelli PM, Morgan JC, Steven A, et al. Fieldcaught Permethrin-resistant Anopheles Gambiae overexpress CYP6P3, a P450 that metabolises pyrethroids. PLoS Genet. 2008;4:e1000286.

25. Ranson H, Cornel AJ, Fournier D, Vaughan A, Collins FH, Hemingway J. Cloning and localization of a glutathione S-transferase class I gene from Anopheles Gambiae. J Biol Chem ASBMB. 1997;272:5464-8.

26. Stevenson BJ, Bibby J, Pignatelli P, Muangnoicharoen S, O'Neill PM, Lian L-Y, et al. Cytochrome P450 6M2 from the malaria vector Anopheles Gambiae metabolizes pyrethroids: sequential metabolism of deltamethrin revealed. Insect Biochem. Mol. Biol. Elsevier. 2011:41:492-502.

27. Smyth GK. Linear models and empirical bayes methods for assessing differential expression in microarray experiments. Stat Appl Genet Mol Biol. 2004;3:3.

28. MacCallum RM, Redmond SN, Christophides GK. An expression map for Anopheles Gambiae. BMC Genomics. 2011;12:620.

29. Nioi P, McMahon M, Ken I, Yamamoto M, Hayes JD. Identification of a novel Nrf2-regulated antioxidant response element (ARE) in the mouse NAD (P) H: quinone oxidoreductase 1 gene: reassessment of the ARE consensus sequence. Biochem J. 2003;374:337-48.

30. Sykiotis GP, Bohmann D. Keap1/Nrf2 signaling regulates oxidative stress tolerance and lifespan in drosophila. Dev Cell Elsevier. 2008;14:76-85.

31. Mathelier A, Fornes O, Arenillas DJ, Chen C, Denay G, Lee J, et al. JASPAR 2016: a major expansion and update of the open-access database of transcription factor binding profiles. Nucleic Acids Res Oxford Univ Press. 2015;44:D110-5.

32. Shannon P, Shannon MP, RUnit S, biocViews GenomicSequence M. Package 'MotifDb'. 2013;
33. Kwak M-K, Itoh K, Yamamoto M, Kensler TW. Enhanced expression of the transcription factor Nrf2 by cancer chemopreventive agents: role of antioxidant response element-like sequences in the nrf2 promoter. Mol Cell Biol Am Soc Microbiol. 2002;22:2883-92.

34. Katsuoka F, Motohashi H, Engel JD, Yamamoto M. Nrf2 transcriptionally activates the MafG gene through an antioxidant response element. J. Biol. Chem. ASBMB. 2005;280:4483-90.

35. Edi CV, Benjamin KG, Jones CM, Weetman D, Ranson H. Multiple-insecticide resistance in Anopheles Gambiae mosquitoes, southern Côte d'Ivoire. Emerg. Infect. Dis. 2012;18:1508-11.

36. Ingham VA, Jones CM, Pignatelli P, Balabanidou V, Vontas J, Wagstaff SC, et al. Dissecting the organ specificity of insecticide resistance candidate genes in Anopheles Gambiae: known and novel candidate genes. BMC Genomics. 2014;15:1018.

37. Chung H, Sztal T, Pasricha S, Sridhar M, Batterham P, Daborn PJ. Characterization of Drosophila Melanogaster cytochrome P450 genes. Proc Natl Acad Sci National Acad Sciences. 2009;106:5731-6.

38. Main BJ, Lee Y, Collier TC, Norris LC, Brisco K, Fofana A, et al. Complex Genome Evolution in A coluzzii associated with increased insecticide usage in Mali. Mol Ecol. 2015;20:5145-57.

39. World Health Organization. Test procedures for insecticide resistance monitoring in malaria vector mosquitoes. WHO; 2013;

40. Liu N, Li M, Gong Y, Liu F, Li T. Cytochrome p450s-their expression, regulation, and role in insecticide resistance. Pestic Biochem Physiol Elsevier. 2015;120:77-81.

41. Puinean AM, Foster SP, Oliphant L, Denholm I, Field LM, Millar NS, et al. Amplification of a cytochrome P450 gene is associated with resistance to neonicotinoid insecticides in the aphid Myzus Persicae. PLoS Genet. 2010;6:e1000999.

42. Harrop TWR, Sztal T, Lumb C, Good RT, Daborn PJ, Batterham P, et al. Evolutionary changes in gene expression, coding sequence and copynumber at the cyp6g1 locus contribute to resistance to multiple insecticides in drosophila. PLoS One. 2014;9:e84879.

43. Rahman MM, Sykiotis GP, Nishimura M, Bodmer R, Bohmann D. Declining signal dependence of Nrf2-MafS-regulated gene expression correlates with aging phenotypes. Aging Cell Wiley Online Library. 2013;12:554-62.

44. Baker DA, Nolan T, Fischer B, Pinder A, Crisanti A, Russell S. A comprehensive gene expression atlas of sex-and tissue-specificity in the malaria vector. Anopheles gambiae BMC Genomics. 2011;12:296.

45. Lycett GJ, McLaughlin LA, Ranson H, Hemingway J, Kafatos FC, Loukeris TG, et al. Anopheles Gambiae P450 reductase is highly expressed in oenocytes and in vivo knockdown increases permethrin susceptibility. Insect Mol. Biol. Wiley Online Library. 2006;15:321-7.

46. Edi CAV, Koudou BG, Bellai L, Adja AM, Chouaibou M, Bonfoh B, et al. Longterm trends in Anopheles Gambiae insecticide resistance in Côte d'Ivoire. Parasit Vectors. 2014;7:500

47. Karim MR, Taniguchi H, Kobayashi A. Constitutive activation of drosophila CncC transcription factor reduces lipid formation in the fat body. Biochem Biophys Res Commun Elsevier. 2015;463:693-8.

48. Sykiotis GP, Bohmann D. Stress-activated cap'n'collar transcription factors in aging and human disease. Sci. Signal. 2010:3:re3.

49. Deng H, Kerppola TK. Regulation of drosophila metamorphosis by xenobiotic response regulators. PLoS Genet. 2013;9:e1003263.

50. Anand A, Crone EJ, Zera AJ. Tissue and stage-specific juvenile hormone esterase (JHE) and epoxide hydrolase (JHEH) enzyme activities and Jhe transcript abundance in lines of the cricket Gryllus Assimilis artificially selected for plasma JHE activity: implications for JHE microevolut. J Insect Physiol Elsevier. 2008;54:1323-31.

51. Wheelock CE, Shan G, Ottea J. Overview of carboxylesterases and their role in the metabolism of insecticides. J Pestic Sci Pesticide Science Society of Japan. 2005;30:75-83.

52. Edi CV, Djogbénou L, Jenkins AM, Regna K, Muskavitch MAT, Poupardin R, et al. CYP6 P450 enzymes and ACE-1 duplication produce extreme and multiple insecticide resistance in the malaria mosquito Anopheles Gambiae. PLoS Genet. 2014;10:e1004236.

53. Voice MW, Kaaz AW, Peet CF, Paine MJ. Recombinant CYP6M2 inhibition by insecticides recommended by WHO for indoor residual spraying against malaria vectors. Drug Metab Rev. 2015;44:56-7.

54. Ranson H, Claudianos C, Ortelli F, Abgrall C, Hemingway J, Sharakhova M V, et al. Evolution of supergene families associated with insecticide resistance. 
Science (80-. ). American Association for the Advancement of Science; 2002;298:179-181.

55. Ngufor C, Mouhamadou C. Ogoma S. AvecNet: AvecNet's contribution to the evidence base for new insecticide based products; 2016.

56. Pimprale SS, Besco CL, Bryson PK, Brown TM. Increased susceptibility of pyrethroid-resistant tobacco budworm (Lepidoptera: Noctuidae) to chlorfenapyr. J Econ Entomol The Oxford University Press. 1997;90:49-54.

57. Hemingway J, Ranson H, Magill A, Kolaczinski J, Fornadel C, Gimnig J, et al. Averting a malaria disaster: will insecticide resistance derail malaria control. Lancet. Elsevier. 2016;387:1785-8.

58. Smyth GK, Michaud J, Scott HS. Use of within-array replicate spots for assessing differential expression in microarray experiments. Bioinformatics. 2005;21:2067-75.

59. MacDonald JW. Affycoretools: functions useful for those doing repetitive analyses with Affymetrix GeneChips. R Packag version. 2008;

60. Huang DW, Sherman BT, Lempicki RA. Systematic and integrative analysis of large gene lists using DAVID bioinformatics resources. Nat. Protoc. Nature Publishing Group. 2008:4:44-57.

61. Shannon P, Markiel A, Ozier O, Baliga NS, Wang JT, Ramage D, et al. Cytoscape: a software environment for integrated models of biomolecular interaction networks. Genome Res. 2003;13:2498-504.

62. Blandin S, Moita LF, Köcher T, Wilm M, Kafatos FC, Levashina EA. Reverse genetics in the mosquito Anopheles Gambiae: targeted disruption of the Defensin gene. EMBO Rep EMBO Press. 2002;3:852-6.

63. Ye J, Coulouris G, Zaretskaya I, Cutcutache I, Rozen S, Madden TL. PrimerBLAST: a tool to design target-specific primers for polymerase chain reaction. BMC Bioinformatics. 2012;13:134.

64. Bustin SA, Benes V, Garson JA, Hellemans J, Huggett J, Kubista M, et al. The MIQE guidelines: minimum information for publication of quantitative realtime PCR experiments. Clin Chem Am Assoc Clin Chem. 2009:55:611-22.

65. Schmittgen TD, Livak KJ. Analyzing real-time PCR data by the comparative CT method. Nat Protoc Nature Publishing Group. 2008:3:1101-8.

66. Huang D, Sherman B, Tan Q, Collins J, Alvord G, Roayaei J, et al. The DAVID gene functional classification tool: a novel biological module-centric algorithm to functionally analyze large gene lists. Genome Biol. 2007;8:R183.

\section{Submit your next manuscript to BioMed Central and we will help you at every step:}

- We accept pre-submission inquiries

- Our selector tool helps you to find the most relevant journal

- We provide round the clock customer support

- Convenient online submission

- Thorough peer review

- Inclusion in PubMed and all major indexing services

- Maximum visibility for your research

Submit your manuscript at www.biomedcentral.com/submit

) Biomed Central 